\title{
INVESTIGATION OF OSCILLATIONS OF HAMMER ROTOR OF GRAIN CRUSHER
}

\author{
Vasiliy Sysuev $^{1}$, Peter Savinyh ${ }^{1}$, Alexey Aleshkin ${ }^{2}$, Semjons Ivanovs ${ }^{3}$ \\ ${ }^{1}$ North-East Agricultural Research Institute, Russia; \\ ${ }^{2}$ Vyatka State University, Russia; ${ }^{3}$ Latvia University of Agriculture, Latvia \\ semjons@apollo.lv
}

\begin{abstract}
Due to the high productivity and the wide range of crushing, rotor crushers are widely used for crushing grain for animals. The paper deals with the movement of the rotor with moving masses as an example of a hammer rotor of the crusher, which can be imagined as a system with several degrees of freedom with nonstationary constraints, taking into account the forces of resistance and nonharmonic periodic external influences. For the generation of differential equations of the movement of the system, we used the Lagrange equations of the second kind, in the solution of which decomposition of the movement according to the proper forms of oscillation was applied. The nature of the change in the external impact was determined experimentally. Distribution of its intensity was estimated by the traces of the crushed particles left on a previously painted lateral surface of the crushing chamber.
\end{abstract}

Keywords: grain crusher, the Lagrange equations of the 2nd kind, kinetic energy.

\section{Introduction}

Grain is a valuable source of feed for farm animals. The body of the animal assimilates more completely nutrients from crushed grain.

Crushing grain is widely used for the preparation of feed for the animals on farms and in the mixed feed industry. For crushing grain, rotary (hammer) crushers are widely used [1-2]. Such crushers have high productivity and provide a wide range of grinding.

During the research of the operation of rotary grain crushers, the main regularities determining the productivity, the degree of crushing, the energy consumption, etc. were revealed.

However, new technological and technical requirements are imposed to the operation of hammer crushers and a process of constant improvement of the structures is underway. Therefore, the development of these machines with justified optimal design parameters is an important and urgent task.

The hammers of the crusher are pivotally connected with the rotor, and, because of uneven feeding of the grain and the resistance forces, they perform some oscillatory movements relative to the point of attachment. Oscillations of the hammers affect the efficiency of the machine. Establishment of theoretical patterns of the oscillation process of hammers allows us to determine the optimal ranges of parameters and make appropriate corrections in the design already at the design stage.

A number of research papers [3-4] have been devoted to the study of the oscillations of pivotally fixed operating tools of agricultural machines; however, theoretical and experimental studies of the grain-hammer interaction that contribute to the improvement of the grain crusher structures are not complete and require refinement.

The aim of this work was a mathematical description of the impact of grain upon the hammer rotor and the determination of the influence of the location of the centre of masses of the hammers upon the process of their oscillations when deviating from the equilibrium movement.

\section{Materials and methods}

For the theoretical study of the process of the grain interaction with the hammer of the crusher, we will compute a design scheme. In contrast to the forces of inertia and restoring forces, the resistance forces have significantly smaller values; therefore, to simplify the design scheme, we assume that the damping will be linear and proportional by the form of the proper oscillations. The basis of the considered mechanical system (Fig. 1) is the rotor 1 of the crusher with pivotally fixed hammer packs $2[5 ; 6]$ on it. The rotation from the electric motor is transmitted through the pulleys and elastic V-belts 3 to the rotor 1 of the crusher, which, in unperturbed movement, has an angular velocity $\omega$. If the rotor deviates from the steady-state rotation according to the law $\omega t$ by a small angle $\varphi_{1}$, an additional pair of forces appears in the V-belts, the moment of which is proportional to the angle $\varphi_{1}$, but opposite to it by direction. 


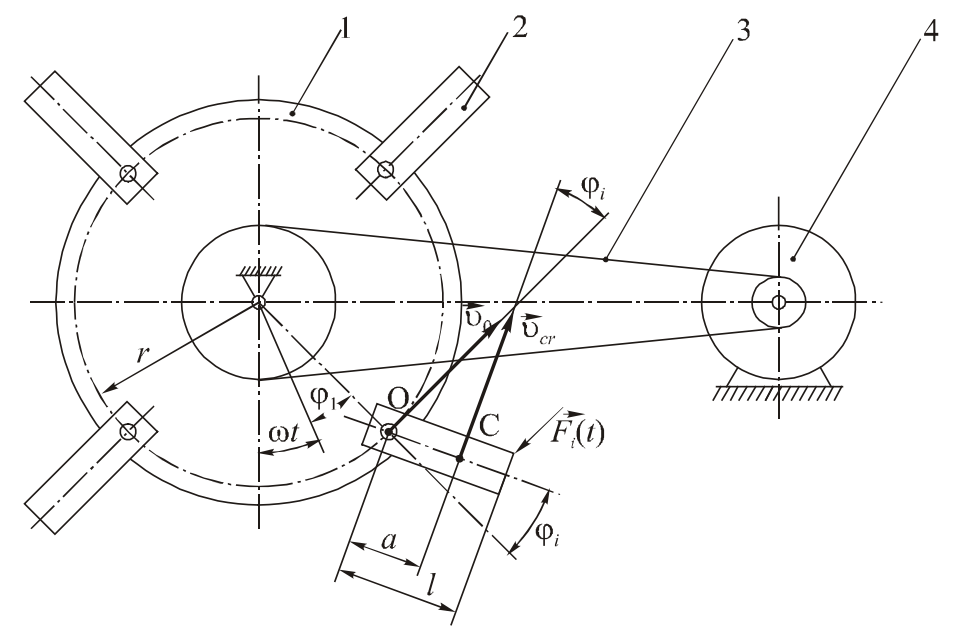

Fig. 1. Scheme of the hammer crusher rotor

The elastic stiffness of the belts $c_{p}$ can be determined by calculation, depending on the type of the belts and the diameter of the pulleys, or experimentally with the electric motor stopped, by applying the known moment to the pulley of the crusher rotor and measuring its small deflection angle. The packs of hammers are located on the rotor uniformly along the circumference of the radius $r$. If the hammers are located in the pack on a common axis, then, when considering a planar circuit, they can be considered as one solid body, pivotally fixed on the rotor. This will reduce the number of degrees of freedom of the system. The angle of deviation of the $i$-th pack of hammers from the radial direction is denoted as $\varphi_{i}$. A non-stationary constraint is imposed on the system, determined by the rotation of the electric motor.

To obtain differential equations of the movement of the system, we use the Lagrange equations of the second kind [7]

$$
\frac{d}{d t}\left(\frac{\partial T}{\partial \dot{\varphi}_{i}}\right)-\frac{\partial T}{\partial \varphi_{i}}=Q_{i}, i=1, \ldots n,
$$

where $T$ - kinetic energy of the system;

$Q_{i}$ - generalised force, corresponding to the $i$-th coordinate;

$\varphi_{1}-$ rotor deviation from the steady-state movement;

$\varphi_{2 \ldots} \varphi_{n}-$ deviation of the hammer packs from the radial direction;

$n$ - number of degrees of freedom of the mechanical system.

These equations can be presented in the matrix form

$$
\frac{d}{d t}\left\{\frac{\partial T}{\partial \dot{\varphi}}\right\}-\left\{\frac{\partial T}{\partial \varphi}\right\}=\{Q\}
$$

where the column vectors correspond to the terms in equation (1).

The kinetic energy of the system $T$ is calculated in the inertial frame of reference as the sum of the kinetic energies of the bodies entering the system [8; 9]

$$
T=\frac{J_{1}\left(\omega+\dot{\varphi}_{1}\right)^{2}}{2}+\sum_{i=2}^{n}\left[\frac{m_{i} v_{0}^{2}}{2}+\frac{J_{i}\left(\omega+\dot{\varphi}_{1}+\dot{\varphi}_{i}\right)^{2}}{2}+m_{i} \cdot \vec{v}_{0} \cdot \vec{v}_{c r i}\right],
$$

where the first term is the kinetic energy of the rotor with the moment of inertia $J_{1}$ relative to the axis of rotation.

Each term of the sum determines the kinetic energy of each pack of hammers, calculated in accordance with the Koenig's theorem: $\vec{v}_{0}$ - speed of the hammer attachment point $\mathrm{O}$ on the rotor, $m_{i}$ - mass of the $i$-th hammer pack, $J_{i}$ - its inertia moment relative to the suspension axis passing 
through the point $\mathrm{O}, \vec{v}_{c r i}$ - relative velocity of the centre of masses of the $i$-th pack of hammers, calculated in the forward moving axes connected with the point $\mathrm{O}$.

We will express the velocities $\vec{v}_{0}$ and $\vec{v}_{c r i}$ in terms of generalised coordinates $\varphi_{i}$ and velocities $\dot{\varphi}_{i}$, while

$$
\begin{gathered}
v_{0}=\left(\omega+\dot{\varphi}_{1}\right) r, \\
v_{c r i}=\left(\omega+\dot{\varphi}_{1}+\dot{\varphi}_{i}\right) a,
\end{gathered}
$$

where $a$-distance from the suspension axis to the centre of masses of the hammers.

The scalar product of these velocities is determined taking into account the angle $\varphi_{i}$ between them:

$$
\vec{v}_{0} \cdot \vec{v}_{c r i}=\left(\omega+\dot{\varphi}_{1}\right)\left(\omega+\dot{\varphi}_{1}+\dot{\varphi}_{i}\right) r \cdot a \cos \varphi_{i} .
$$

Let us expand the function $\cos \varphi_{i}$ into a Maclaurin series and confine ourselves to the terms of the second order of smallness:

$$
\cos \varphi_{i} \approx 1-\frac{\varphi_{i}^{2}}{2}
$$

By substituting expressions (4), (5), (6) into (3), we obtain

$$
T=\frac{J_{1}\left(\omega+\dot{\varphi}_{1}\right)^{2}}{2}+\sum_{i=2}^{n}\left[\begin{array}{l}
\frac{J_{1}\left(\omega+\dot{\varphi}_{1}+\dot{\varphi}_{i}\right)^{2}}{2}+\frac{m_{i} r^{2}\left(\dot{\varphi}_{1}+\omega\right)^{2}}{2}+ \\
+m_{i} \operatorname{ar}\left(\omega+\dot{\varphi}_{1}\right)\left(\omega+\dot{\varphi}_{1}+\dot{\varphi}_{i}\right)\left(1-\frac{\varphi_{i}^{2}}{2}\right)
\end{array}\right] .
$$

Let us calculate the derivatives on the left-hand side of the first Lagrange equation of the second kind and restrict ourselves to the first-order quantities of smallness in the obtained expressions

$$
\frac{d}{d t}\left(\frac{\partial T}{\partial \dot{\varphi}_{1}}\right)-\frac{\partial T}{\partial \varphi_{1}}=\ddot{\varphi}_{1}\left(J_{1}+\sum_{i=2}^{n}\left(J_{i}+m_{i} r^{2}+2 m_{i} a r\right)\right)+\sum_{i=2}^{n} \ddot{\varphi}_{i}\left(J_{i}+m_{i} a r\right) .
$$

For all other Lagrange equations (1) $(i=2 \ldots n)$, the left-hand side takes the form

$$
\frac{d}{d t}\left(\frac{\partial T}{\partial \dot{\varphi}_{i}}\right)-\frac{\partial T}{\partial \varphi_{i}}=\ddot{\varphi}_{1}\left(J_{1}+m_{i} a r\right)+\ddot{\varphi}_{i} J_{i}+m_{i} a r \omega^{2} \varphi_{i} .
$$

We will introduce designations for the constant values $D, L, K$ :

$$
\left\{\begin{array}{l}
D=J_{1}+\sum_{i=2}^{n}\left(J_{i}+m_{i} r^{2}+2 m_{i} a r\right) ; \\
L=J_{i}+m a r ; \\
K=m_{i} \operatorname{arc}^{2} .
\end{array}\right.
$$

The Lagrange equation of the second kind in the matrix form (2) can be written in the form:

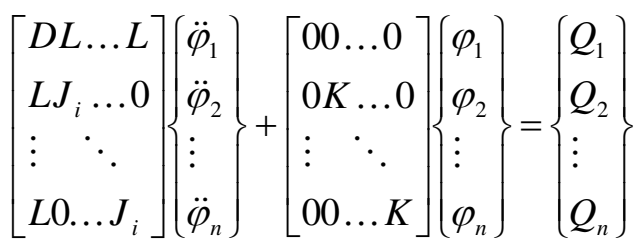

The column vector of the generalised forces $\{Q\}$ can be presented as the sum of the components 


$$
\{Q\}=-[B]\{\dot{\varphi}\}-\left\{Q_{y n}\right\}+\{Q(t)\},
$$

where $[B]-$ damping matrix,

$\{\dot{\varphi}\}-$ column vector of the generalised velocities;

$$
\begin{aligned}
& \left\{Q_{y n}\right\}=+\left\{\begin{array}{l}
c_{p} \varphi_{1} \\
0 \\
\vdots \\
0
\end{array}\right\} \begin{array}{l}
- \text { vector-column of the generalised forces from the elastic forces of } \\
\text { the rotor drive belts; }
\end{array} \\
& \{Q(t)\}=-\left\{\begin{array}{l}
\sum_{i=2}^{n}(r+l) F_{i}(t) \\
F_{2}(t) \cdot l \\
\vdots \\
F_{n}(t) \cdot l
\end{array}\right\} \begin{array}{l}
\text { - impact forces upon the system from the side of the } \\
\text { material to be crushed. }
\end{array}
\end{aligned}
$$

The dependencies $F_{i}(t)$ are tabulated according to the experimental data, the forces $F_{i}(t), i=2 \ldots n$ having a periodic nature of the change (Figure 2); they are identical in appearance, but are phaseshifted, depending on the location of the hammer packs along the circumference of the rotor and the material feeding zone [10]. So, for four packets $(n=5)$, the phase offset is an angle equal to $\pi / 2$.

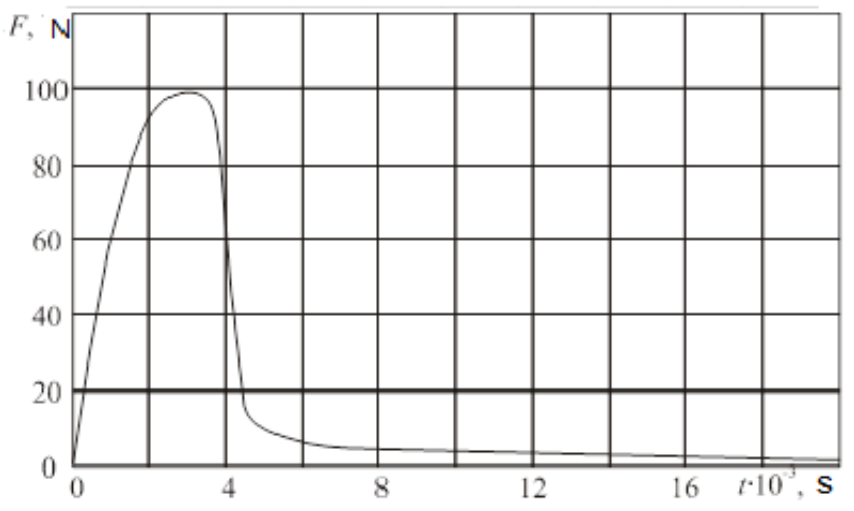

Fig. 2. Experimental dependence of the force $F(t)$ on time

Moving all the terms containing the generalised coordinates and velocities to the left-hand side of the equation (12), we obtain

$$
[M]\{\ddot{\varphi}\}+[B]\{\dot{\varphi}\}+[C]\{\varphi\}=\{Q(t)\},
$$

where $[B]-$ damping matrix;

$$
\begin{aligned}
& {[M]=\left[\begin{array}{l}
D L \ldots L \\
L J_{i} \ldots 0 \\
\vdots \\
L 0 \ldots J_{i}
\end{array}\right]-\text { matrix of masses; }} \\
& {[C]=\left[\begin{array}{ll}
c_{p} & 0 \ldots 0 \\
0 & K \ldots 0 \\
\vdots & \ddots \\
0 & 0 \ldots K
\end{array}\right] \text { - matrix of stiffnesses. }}
\end{aligned}
$$

With the assumption of proportional damping according to the forms of proper oscillations, this matrix is a linear combination of the matrices of masses and stiffnesses

$$
[B]=a[M]+d[C] \text {, }
$$


where $a, d$-constant proportionality coefficients.

Besides, the matrix equation (14) can be transformed to the principal coordinates $-u_{i}$, and the equation for each of the principal coordinates can be solved independently [6].

Let us find the proper (natural) frequencies and forms of oscillations of the system, considering the equation of free oscillations

$$
[M]\{\ddot{\varphi}\}+[C]\{\varphi\}=0 .
$$

A particular solution of this equation has the form

$$
\{\varphi\}=\{A\} \sin p t .
$$

Substituting it into the equation (10) and equating the coefficients at sinpt, we obtain

$$
\left[-p^{2}[M]+[C]\right]\{A\}=0 .
$$

We will designate in this equation the so-called characteristic matrix $[H]=,\left[-p^{2}[M]+[C]\right]$. Then the equation (11) takes the form $[H]\{A\}=0$. The determinant of the matrix $[H]$, equated to zero, is the equation of frequencies: $|H|=0$. The roots of this equation $p_{i}^{2}$ are squares of proper (natural) frequencies of oscillations of the system. The eigenvectors (proper vectors) of the matrix $[C]^{-1}[M]$, determined with exactness up to a constant factor, are the proper forms (eigenmodes) of the system oscillations. The totality of columns $\{A\}_{i}$, united into a square matrix in the order of an increasing value of the corresponding frequencies, is a matrix of proper forms (eigenmodes) of oscillations

$$
[A]=\left[\{A\}_{1}\{A\}_{2} \ldots\{A\}_{n}\right] .
$$

For practical calculations of $\lambda_{i}=1 / p_{i}^{2}$ and $\{A\}_{i}$, there can be applied standard calculation subprogrammes of the proper values (eigenvalues) and proper vectors (eigenvectors) of a square matrix $[C]^{-1}[M]$ in the algorithmic language "Fortran": NROOT and EIGEN, which are based on the Jacobi method and work together.

After the matrix of the forms is determined, we transform the equation (14), using the orthogonality property of the matrix $[A]$. Let us multiply its both sides by the transposed matrix of forms $[A]^{T}$, and each term on the left-hand side by the unit matrix $[E]=[A][A]^{-1}$ :

$$
[A]^{T}[M][A][A]^{-1}\{\ddot{\varphi}\}+[A]^{T}[B][A][A]^{-1}\{\dot{\varphi}\}+[A]^{T}[C][A][A]^{-1}\{\varphi\}=[A]^{T}\{Q(t)\} .
$$

In this equation we denote:

1. $\left[M_{\Gamma}\right]=[A]^{T}[M][A]=\left[\backslash m_{\Gamma i} \backslash\right]-$ main matrix of masses, which due to the orthogonality of the proper forms (eigenmodes) of oscillations, is diagonal, besides: $m_{\Gamma i}=(A)_{i}[M]\{A\}_{i}$;

2. $\left[C_{\Gamma}\right]=[A]^{T}[C][A]=\left[\backslash c_{\Gamma i} \backslash\right]-$ main matrix of stiffnesses, also diagonal, and $c_{\Gamma i}=(A)_{i}[C]\{A\}_{i}$;

3. $\left[B_{\Gamma}\right]=[A]^{T}[B][A]$ - main damping matrix; taking into account the proportionality condition, this matrix is also diagonal $\left[B_{\Gamma}\right]=\left[\backslash B_{\Gamma i} \backslash\right]$, but $\beta_{\Gamma i}=a(A)_{i}[M]\{A\}_{i}+d(A)_{i}[C]\{A\}_{i}$;

4. $\{u\}=[A]^{-1}\{\varphi\}-$ column vector of the main generalised coordinates $u_{i}$ and the vector column of their derivatives: $\{\dot{u}\}=[A]^{-1}\{\dot{\varphi}\},\{\ddot{u}\}=[A]^{-1}\{\ddot{\varphi}\}$;

5. $\left\{Q_{\Gamma}(t)\right\}=[A]^{T}\{Q(t)\}-$ column vector of the main generalised forces, where $Q_{\Gamma i}(t)=\{A\}_{i}^{T}\{Q(t)\}=A_{1 i} Q_{1}(t)+\ldots+A_{n i} Q_{n}(t)$; in a tabular presentation of arrays with a definite time step, the functions $Q_{\Gamma i}(t)$ will also be defined as arrays $Q_{i}(t)$ of discrete values with the same time step.

In accordance with the notation introduced, the system of equations (20) takes the form

$$
\left[M_{\Gamma}\right]\{\ddot{u}\}+\left[B_{\Gamma}\right]\{\dot{u}\}+\left[C_{\Gamma}\right]\{u\}=\left\{Q_{\Gamma}(t)\right\} .
$$

The matrix equation (13) splits into separate equations of the form

$$
m_{\Gamma i} \ddot{u}_{i}+\beta_{\Gamma i} \dot{u}_{i}+c_{\Gamma i} u_{i}=Q_{\Gamma i}(t) \text {. }
$$


By dividing each of the equations (1.1.14) by $m_{\Gamma i}$ we obtain

$$
\ddot{u}_{i}+\frac{{ }^{{ }} \Gamma i}{m_{\Gamma i}} \dot{u}_{i}+\frac{c_{\Gamma i}}{m_{\Gamma i}} u_{i}=\frac{Q_{\Gamma i}(t)}{m_{\Gamma i}} .
$$

Let us denote $\frac{c_{\Gamma i}}{m_{\Gamma i}}=p_{i}^{2}$ - square of the proper (natural) frequencies of oscillations, $\frac{{ }^{6} \Gamma i}{m_{\Gamma i}}=2 n_{i}$ - coefficient that takes into account the forces of viscous resistance. At a small resistance, it is assumed that $n_{i}=\gamma_{i} p_{i}$, where $\gamma_{i}$-damping coefficient [6] $0 \leq \gamma_{i} \leq 0.3$. In this case we write the equation (23) as

$$
\ddot{u}_{i}+2 n_{i} \dot{u}_{i}+p_{i}^{2} u_{i}=\frac{Q_{\Gamma i}(t)}{m_{\Gamma i}} .
$$

The solution of this equation will be determined with the help of the Duhamel integral

$$
u_{i}=\left(C_{1 i}^{*} \cos p_{i}^{*} t+C_{2 i}^{*} \sin p_{i}^{*} t\right) e^{-n_{i} t}+\int_{0}^{t} \frac{Q_{\Gamma i}(\tau)}{m_{\Gamma i} p_{i}^{*}} e^{-n_{i}(t-\tau)} \sin p_{i}^{*}(t-\tau) d \tau,
$$

where $p_{i}^{*}=\sqrt{p_{i}^{2}-n_{i}^{2}}, C_{1 i}^{*}, C_{2 i}^{*}-$ arbitrary constants of integration, determined by the initial conditions.

After determining the time series for $\{u\}$, let us proceed to the original generalised coordinates

$$
\{\varphi\}=[A]\{u\} \text {. }
$$

\section{Results and discussion}

According to the above algorithm, programs for the determination of generalised coordinates $\{\varphi\}$ are compiled using standard subprograms to find the proper (natural) frequencies and forms of the system oscillations. As an example, let us consider two variants of the mass distribution of the hammer rotor (Table 1) and compare the behaviour of these systems in time. Both rotors had four packs of hammers, that is $n=5$.

Table 1

Initial data for the calculation of the mass distribution of the hammers of the crusher rotor

\begin{tabular}{|l|c|c|}
\hline \multicolumn{1}{|c|}{$\begin{array}{c}\text { Values characterizing the distribution of masses } \\
\text { and stiffnesses of the system }\end{array}$} & \multicolumn{2}{c|}{ Initial data for calculations } \\
\cline { 2 - 3 } & Variant 1 & Variant 2 \\
\hline Radius of the location of the suspension axes on the rotor $\mathrm{r}, \mathrm{m}$ & 0.17 & 0.17 \\
\hline $\begin{array}{l}\text { Distance from the axis of suspension to the centre of masses of the } \\
\text { hammer } a, \mathrm{~m}\end{array}$ & $39 \cdot 10^{-3}$ & $22 \cdot 10^{-3}$ \\
\hline $\begin{array}{l}\text { Distance from the suspension axis to the point of application of the } \\
\text { resultant external periodic force } l, \mathrm{~m}\end{array}$ & 0.060 & 0.074 \\
\hline Mass of the pack of hammers $m_{i}, \mathrm{~kg}$ & 1.422 & 2.430 \\
\hline Velocity of the hammer end in unperturbed movement, $\mathrm{m} \mathrm{s}^{-1}$ & 63.4 & 63.4 \\
\hline \multicolumn{2}{|c|}{ Coefficients in matrices $[M]$ and $[C]$ of the equation $(9):$} \\
\hline$D, \mathrm{~kg} \cdot \mathrm{m}^{2}$ & 0.65 & 0.77 \\
\hline$L, \mathrm{~kg} \cdot \mathrm{m}^{2}$ & 0.0120 & 0.0127 \\
\hline$J_{i}, \mathrm{~kg} \cdot \mathrm{m}^{2}$ & 0.0026 & 0.0039 \\
\hline$c_{p}, \mathrm{~N} \cdot \mathrm{m}$ & 4038 & 4038 \\
\hline$K, \mathrm{~N} \cdot \mathrm{m}$ & 721 & 600 \\
\hline
\end{tabular}

The first variant differs from the second by the fact that the centre of gravity of the hammer packs is displaced from the suspension axis due to the location of additional masses closer to the working ends of the hammers. In addition, the mass of the pack of such hammers is less than that in the second variant of the hammer of parallelepipedal shape with an equal width of the zone of impact upon the 
material. As a result of calculations, the following frequency spectra $p_{i}$ were obtained: Variant 1: 78.5; 526.6; 526.6; 526.6; $651.1 \mathrm{rad} \cdot \mathrm{s}^{-1}$; Variant $2: 72.1 ; 394.8 ; 394.8 ; 394.8 ; 448.0 \mathrm{rad} \cdot \mathrm{s}^{-1}$. Only three frequency values are different, the others are multiple. With a symmetrical arrangement of the hammer packs on the rotor, this regularity is retained also for any other number of the freedom degrees of the system at $n \geq 3$. If the rotor of the crusher rotates without a load, then the generalized coordinates $\varphi_{i}$ are equal to zero $\varphi_{i 0}=0$. Figure 3 shows the change in the coordinate $\varphi_{1}-$ deviation of the rotor at a sudden impact of the material. Figure 4 depicts the changes in the coordinate $\varphi_{2}$ per rotor revolution with a steady-state movement and periodic impact of the material upon each of the hammer packs. As the first line of the matrix of shapes $[A]$ shows, the oscillations of the rotor occur under the impact of both the first and second main oscillations, to which the frequencies $p_{1}$ and $p_{n}$ correspond, where the first main oscillation has a more essential value.

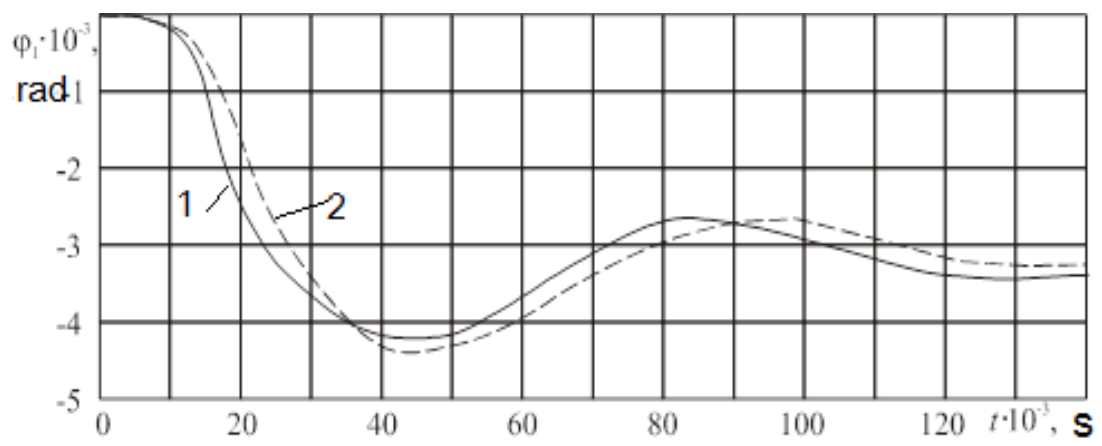

Fig. 3. Dependences of the change in the coordinate $\varphi_{1}$ after the beginning of the material supply on time: 1 - according to the first variant; 2 - according to the second variant

The movement of the hammers is described by oscillations that are composed of the changes in the main coordinates, which have the whole spectrum of proper (natural) frequencies. The decisive is the movement of the main coordinate with the second proper (eigen) frequency. Redistribution of the mass of hammers from the suspension axis to the ends (Variant 1) entails an increase in the components of the matrix of stiffnesses $[C]$ and the values of the natural frequencies. Maximum deviation of the hammers per one revolution of the rotor (Fig. 4) from the unperturbed movement of the hammers in the first variant is slightly less, as is the recovery time to the zero level.

In this case, there is also a slight decrease in the amplitude of the oscillations of the generalised coordinates and the recovery time for the rotor according to the first variant (Fig. 4).

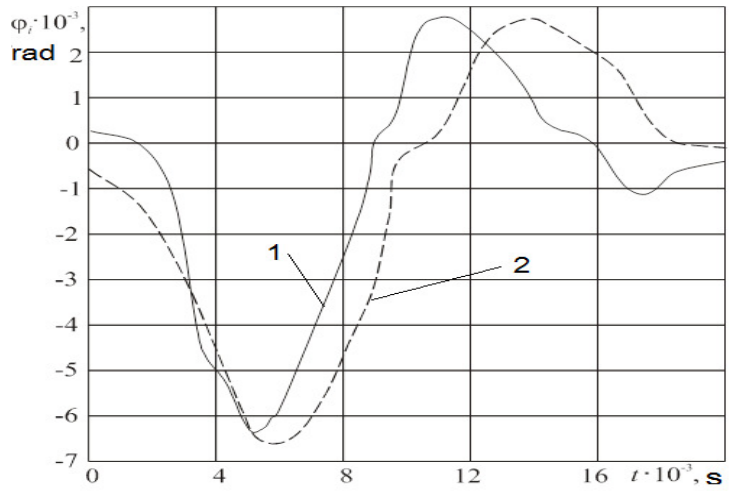

Fig.4. Dependences of the change in the coordinate $\varphi_{i}$ on time with the steady-state movement of the rotor: 1 - according to the first variant; 2 - according to the second variant

This reduces the value of the energy dissipated during the movement of the system, and, consequently, the energy for the drive of the crusher rotor, which is expedient. As it is evident from the analysis of the expansion of the movement of the system according to the proper forms of oscillations, the decisive values for changing the generalised coordinates are the values of the proper (natural) frequencies of oscillations. The frequency of external influences is plotted along the abscissa axis. On the ordinate axis $-K_{i}$ for $=1,2,3$, of which: $K_{1}-$ gain factor for the deviation angle of the rotor $\varphi_{1} ; K_{2}-$ for the deviation angle of the hammer, to which a single periodic impact $\varphi_{2}$ is applied; $K_{3}$ 
- gain factor for the deviation angle of the hammer, to which no such external impacts $\varphi_{3}$ are directly applied. Analysis of the calculated two variants shows that at a rotational velocity of the rotor $v=\omega=260-270 \mathrm{rad} \cdot \mathrm{s}^{-1}$ the oscillation amplitudes of all generalised coordinates are smaller for the rotor with hammers with displaced masses (Variant 1 Table 1). In order to make a quantitative estimation of the deviations of generalised coordinates for specific conditions (structures), it is necessary to carry out numerical calculations for the expressions $(25,26)$. As a result of the analysis of these equations, the displacement of the hammer mass to the periphery of the crushing chamber was recommended. However, when designing rotor crushers, the mass of hammers cannot be redistributed arbitrarily. It is also necessary to have a certain combination of its dimensions and an axis of suspension, ensuring its balance to impact.

Thus, the method described here allows to determine the deviation of the selected generalised coordinates of a mechanical system from the steady-state movement and to evaluate the effect of structural and technological factors on the maximum values of these deviations.

\section{Conclusions}

1. The described method allows to determine the deviation of the selected generalised coordinates of a mechanical system from the steady-state movement and to assess the impact of the design and technological factors on the maximum values of these deviations.

2. At a rotor speed of $v=\omega=260-270 \mathrm{rad} \cdot \mathrm{s}^{-1}$, the oscillation amplitudes of all generalised coordinates are smaller for the rotor with hammers with displaced masses. In order to make a quantitative estimation of the deviations of generalised coordinates for specific conditions (structures), it is necessary to carry out numerical calculations for the obtained expressions.

3. It is preferred to displace the hammer mass to the periphery of the crushing chamber. However, when designing this unit, it should be taken into account that there must be a certain size of the hammer and the suspension axis, which ensures its balance to impact.

\section{References}

1. Сысуев В., Алёшкин А., Савиных П. Кормоприготовительные машины. (Forage Preparation Machines), vol. 1., Kirov, 2009. 639 p. (In Russian).

2. Sysuev, V., Ivanovs, S., Savinyh, P., Kazakov, V. Movement and transformation of grain in twostage crusher Engineering for Rural Development, Proceedings, Vol.14, 2015. pp. 22-27.

3. Vaculik, P., Maloun, J., Chladek, L., Poikryl, M. Disintegration process in disc crushers. Research in Agricultural Engineering. Vol. 59 (3), 2013, pp. 98-104.

4. Sysuev, V., Savinyh, P., Aleshkin, A., Ivanovs, S. Simulation of elastic deformation propagation of grain under impact crushing in crusher. Engineering for Rural Development, Proceedings, Vol. 15, 2016. pp.1065-1071.

5. Dreizler, R.M., Ludde C.S. Theoretical Mechanics. Springer, 2010, 402 p.

6. Алешкин А. Механические колебания в процессах кормоприготовления для животноводства (Mechanical oscillations in feed preparation for livestock). Kirov, 1999. 73 p. (In Russian).

7. Сысуев В., Алешкин А., Савиных П., Халтурин В. Построение амплитудно-частотной характеристики молоткового ротора дробилки (Constructing an amplitude-frequency characteristic of the hammer rotor of the crusher). Технические средства для ресурсосберегающих технологий в растениеводстве и животноводстве (Technical means for resource-saving technologies in crop production and animal husbandry). Kirov, 1997, pp. 123-134. (In Russian).

8. Young D.H., Weaver W. Oscillations in engineering, 1985. $472 \mathrm{p}$.

9. Алешкин А. Исследование колебаний молоткового ротора дробилки методом главных координат (Investigation of oscillations of the hammer rotor of the crusher by the method of principal coordinates). Деп. в ВИНИТИ: № 1549-В98. Moscow, 1998. 13 p. (In Russian).

10. Халтурин В. Совершенствование конструктивных и технологических параметров молотковой дробилки зерна с колосниковой решёткой (Improvement of structural and technological parameters of a grain hammer crusher with a grate), Kirov, 1998. 196 p. (In Russian). 